تأثير حامض الجبريليك على خصائص الإبات ونمو وقوة البادرة تحت ظروف الإجهاد الملحي في الذرة (Zea mays L) الثامية

\author{
فاطمة فرج محمد، احمد سالم بو هدمة
}

حبوب الأرة الشامية صنف هجين ثلاثي .اب بحامض

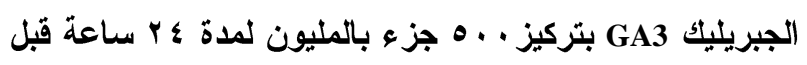

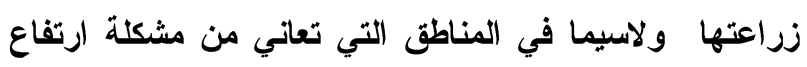

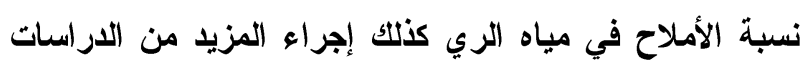

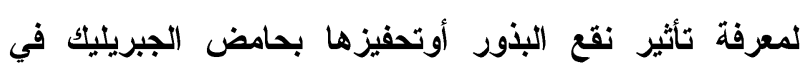
صفات النمو والحاصل للأرة تحت تأثير الإجهاد الملحي لماء لماء

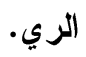
الكلمات المفتاحية: الملوحة، قوة البذرة، متوسط زمن

الإنبات، حامض الجبريليك، الأرة الشامية.

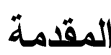

تعد الملوحة في المناطق الجافة وشبه الجافة إحدى أهم

المشاكل التي تؤثر سلبا في نمو وتطور النبات لاسيما فـي مرحلة الإنبات ونمو البادرة ويعتبر الإجهاد الملحي من أهم

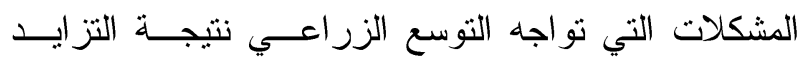

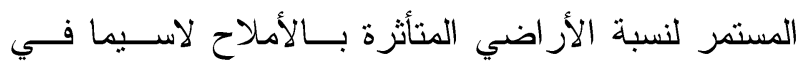
المناطق المروية بسبب الاستخدام المفرط لمياه الري وعدم

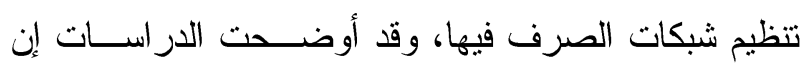

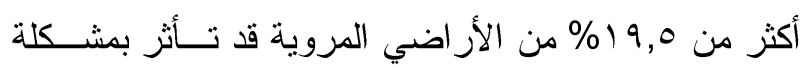

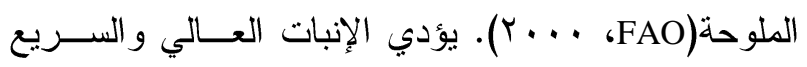

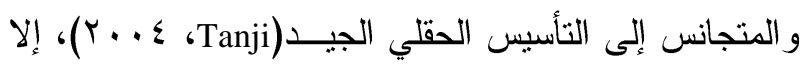

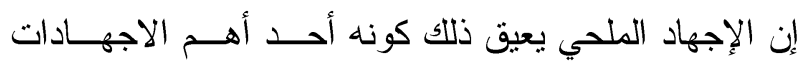
الفسيولوجية التي تؤثر في إنبات البذور ونمو البادرة والذي بدوره يؤثر في مر احل النمو اللاحقة نتيجة تجمع أوتــر اكم

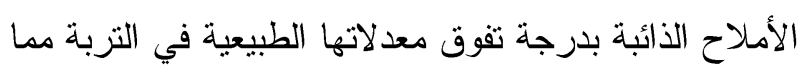

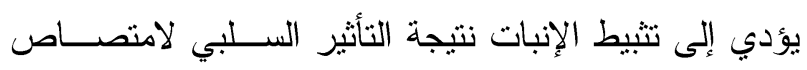

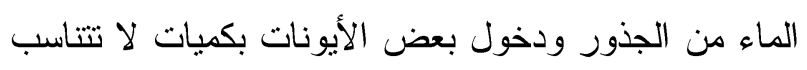

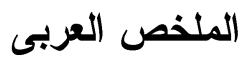

أجريت التجربة بمعمل تقنية الحبوب قمم المحاصيل -

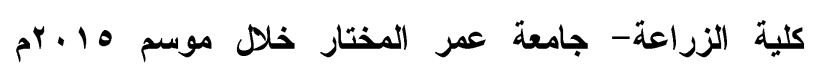

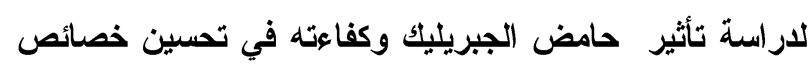
الإببات ونمو البادرة تحت تأثير الإجهاد الملحي في الأرة

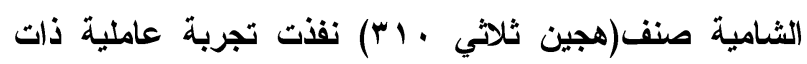

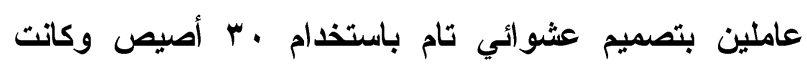

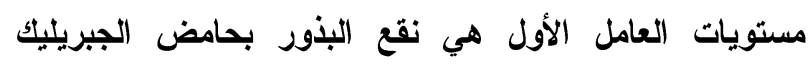

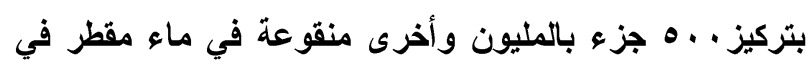

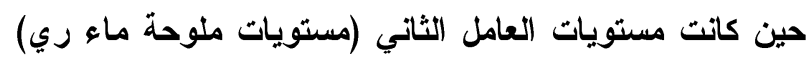

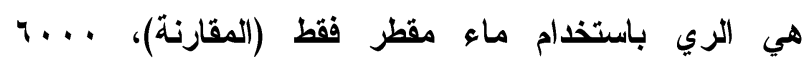

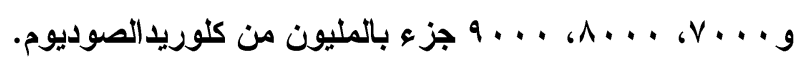
وقد أظهرت النتائج تفوق البذور المنقوعة بحامض

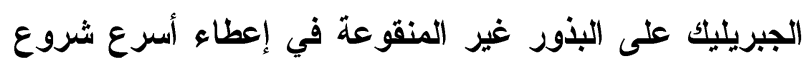
للإنبات(الانبثاث) وأعلى المتوسطات لنسبة وقوة الإنبرة الإبات

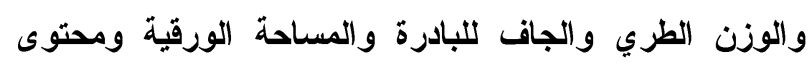
الكلوروفيل. - الكوزن الطزي

أما بالنسبة لتركيزات NaCl فقد أعطى وسط الماء المقطر فقط)(المقارنة(أسرع شروع وأعلى المتوسطات لنسبة الإنبات كما لوحظ سرعة تاخير الانبثاث وانخفاض نسبة الإنبات بزيادة ولثرة الإنيات تركيزات كلوريد الصوديوم حتى . . . 9 جز ع بالمليون NaCl.

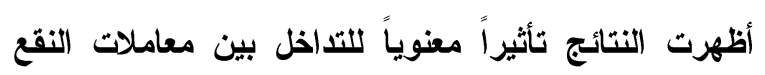
ومستويات الملوحة المستخدمة في أغلب الصفات المدروسة

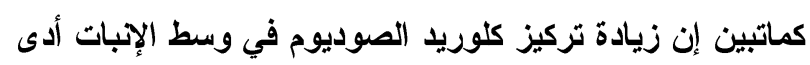

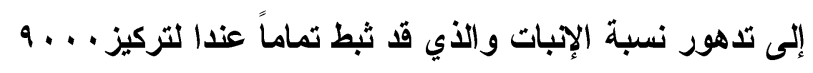

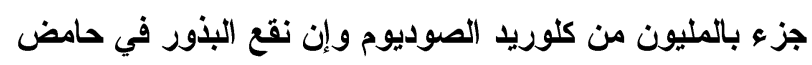

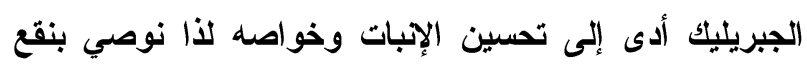

'قسم المحاصيل كلية الزراعة-جامعة عمر المختار - البيضاء- ليبيا Fatmaalzhra84@yahoo.com

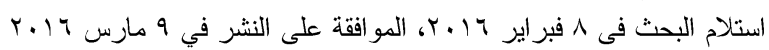


بعض التغيرات لجميع النباتات نظرا لدوره في زيادة الضغط الأسموزي الخلوي (أبوزيد، ، 99 (1). تهدف هذه الدراسة إلى معرفة تأثير النقع في حامض الجبريليك ومدى كفاءته في تحسين خصائص الإنبات ونمو البادرة تحت تأثير الإجهاد الملحي لماء الري في الذرة

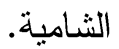

\section{المواد وطرق البحث}

أجريت التجربة بمعمل تقنية الحبوب قم المحاصــيل (كلية الزر اعة/ جامعة عمر المختار) خلال الموسم الصيفي 1 م rم بتصميم عشو ائي تام للتجارب العاملية وفي أصص بلاستيكية حيث كررت كل معاملة س مـرات وكــان كـلـ أصيص بحتوي على ؛ كغم من التربة بعد غســلها جيــــا

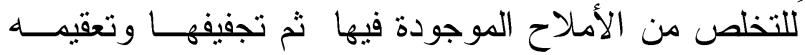
تحت أثنعة الثمس لمدة ؟ كاعة لمعرفة تأثنبر نقع البذور بحامض الجبريليك GA3 بتركيز · . مجزء بالمليون لمدة؛ ساعة وأخرى منقوعة في ماء مقطر لنفس الفترة في إنبات

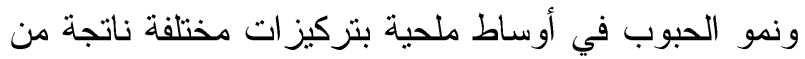
إذابة كميات من كلوريدالصوديوم

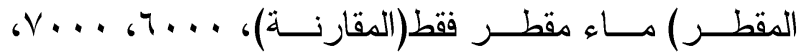

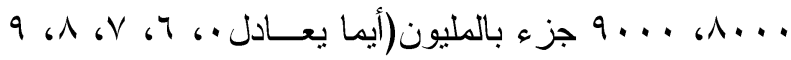
جم/لتز ومدى كفاءته في تحسين خصائص الإنبات ونمــو

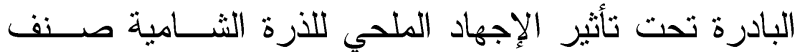
(هجين ثلاثي · (ب) ونم اخذ القياسات لمرحلة نمو البادرات

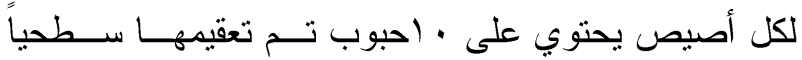
بمحلول هاييوكلورات الصوديوم بتركيزات 0\%

\section{الصفات المدروسة}

1-عدد الأيام من الزر اعة إلى بدء الانبناق طبقا لما ذكــره

.(Shonjani. 2002)

r-العد النهائي لنسبة الإنبات(\%) يقاس بعد انتهــاء مـــدة

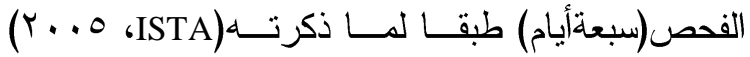
وذللك بحساب العدد الكلي للبادرات الطبيعية بعد V يوم

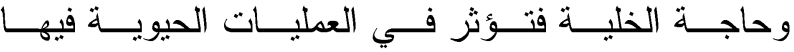
Tsakalidi) في مرحلة الإنبات هي عامل مهم لان الملوحة غالبا ماتكون $\ddot{a}$

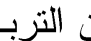
طحية مب مبن الس

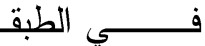
Gajid) المحاصيل الحساسة للملوحة في المر احل المبكرة خصوصاً عندا لري بالماء المالح ولكنها يمكن أن تتحمـلـل الإجهــاد الملحي في المر احل المتأخرة (Carpici و آخــرون، و ه. . ب)

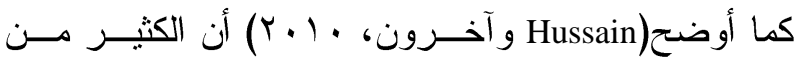
الأنواع النباتية و التي تتضمن معظم المحاصيل يتنبط نموها تحت الظروف الملوحة المرتفعة وأن الإجهاد الملحي يثـبط نمو النباتات ليس فقط بسبب التأثنر الاسموزي لانتقال الماء

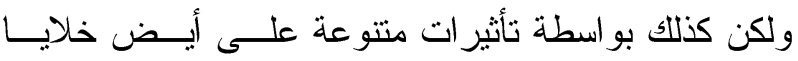
النبات، كما وجد(Carpici و آخرون، 9 9. . Y)، عند استخدامه

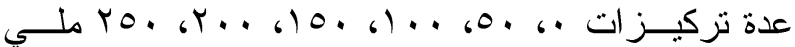
مولار من كلوريد الصوديوم أن زيادة نركيز الأملاح يؤدي إلىى انخفاض نسبة الإنبات ودليل الإنبات وكــذلك الــوزن الجاف للبادرة، وكانت اعلي نسبة انخفــاض عنــدالتركيز

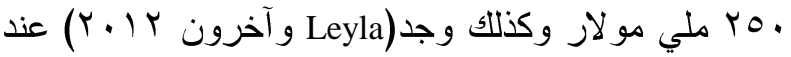

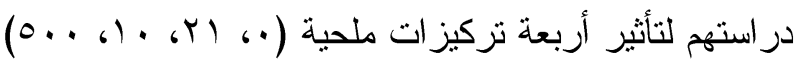
ملي مولار من كلوريد الصوديوم أن زيادة تركيز الأمسـلاح يؤدي إلى تأثيرات سلبية في سرعة الإنبات ومعدل الإنبات ودليل قوة الإنبات وطول الجذيروطول الريشة وطول غمد

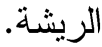

تؤدي الهرمونات النباتية دوراً مهماً في إنبات البذور إذ إذ يتطلب إنبات البذرة نظاماً إنزيميا فعالاً للقيام بعمليتي البناء و الهدم في أثناء عملية الإنبات ويعتبر حامض الجبريليك أحد أهم هذه الهرمونات الذي يؤدي إلى زيادة سرعة الإنبات من خلال تحفيز إنزيمات التحلل المائي الضرورية لتحليل المواد الغذائية وانقسام الخلايا كالألفا أميليز وبيتا أميليز، فضلاً عن عدد من الإنزيمات أهمها البروتيز الريبونيوكليز(Attiya و Jaddoa، (1) كما أنها تسبب 
مجلة الإسكندرية للتبادل العلمى - (مجلد Tr العدد () يناير - مارس 17 •

\section{النتائج والمناقشة}

1- تأثير حامض الجبريليك GA3

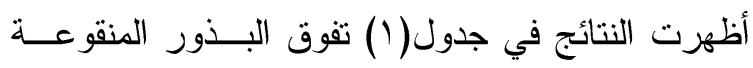
بحامض الجبريليك على البذور غير المنقوعة في إعطــاء

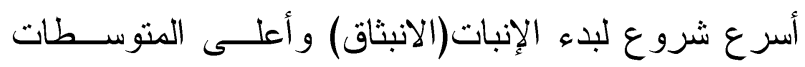

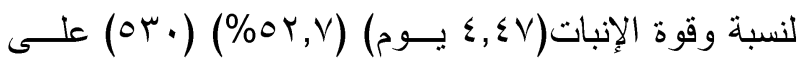

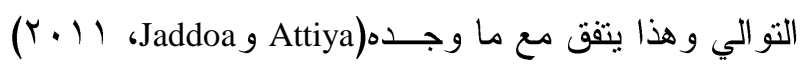

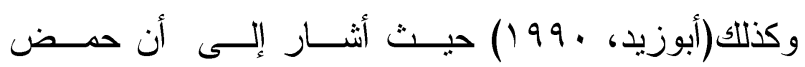

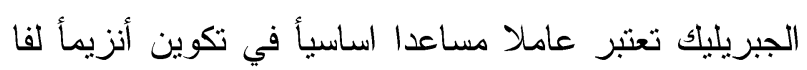

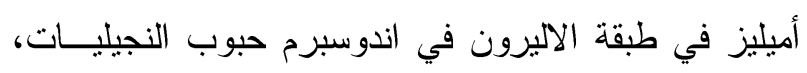

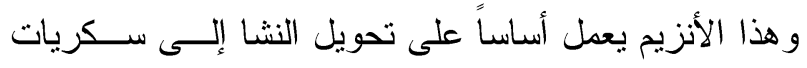

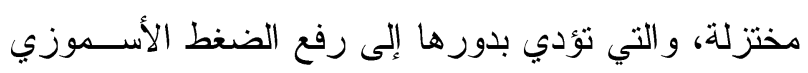

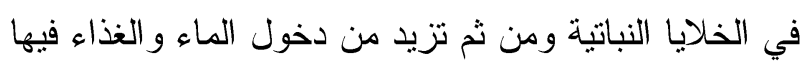
مما يتسبب في انتفاخها وكبر حجمها.

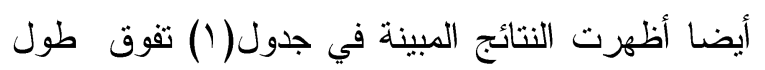
البادرات وكنلك طول الجذير الناتجة من الحبوب المنقوعة

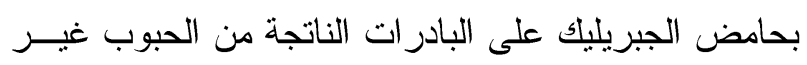

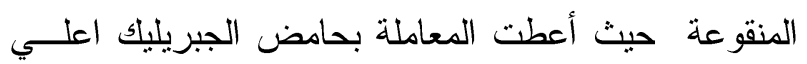

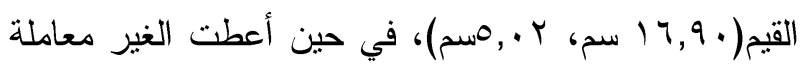

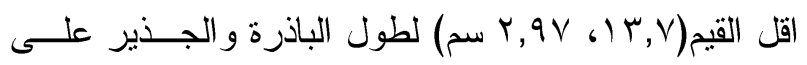

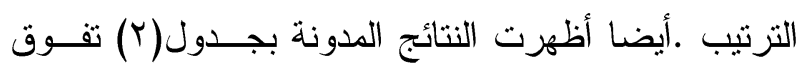

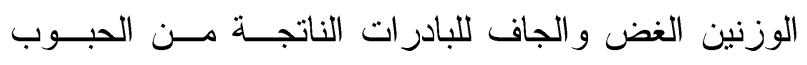

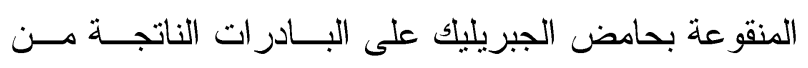

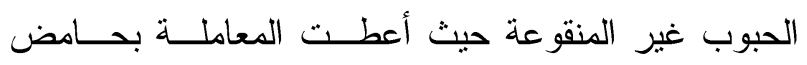

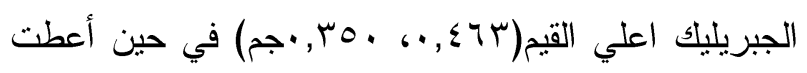

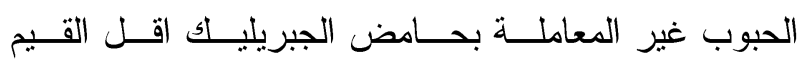

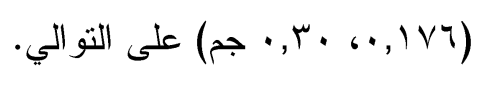

كذلك تقوقت معاملة النقع بحامض الجبريليك معنويا في

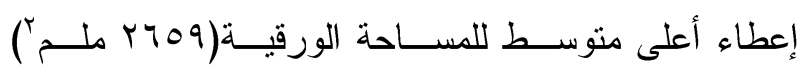

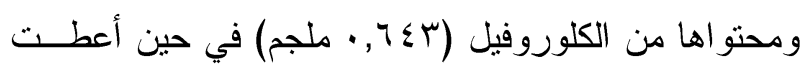

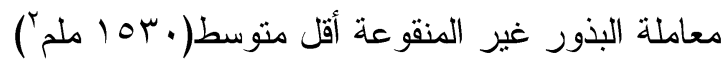

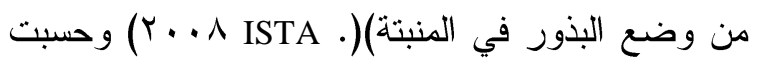

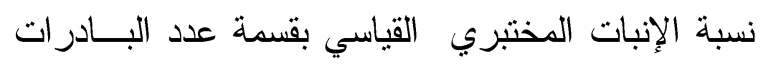

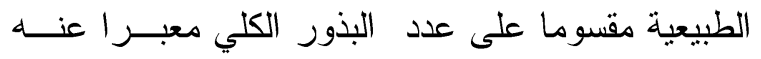
كنسبة مئوية تم حساب نسبة الإنبات مع الصيغة التالية التالية:

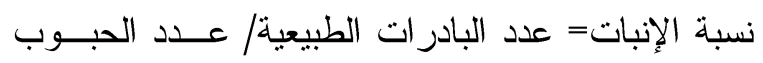
الكلي × ... r-طول الجذير والريشة(سم) في فحص الإنبات المختبري

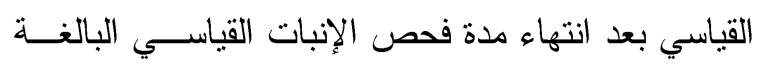

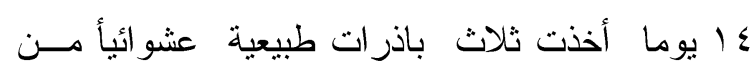
كل أصيص وتم قياس طول الجذير بعد فصله من نقطة اتصاله بالبذرة و الريشة بعد فصلها من نقطة اتصــالها بالسويقة الجنينية الوسطى(AOSA، بـ (1919).

ع-الوزن الغض والجاف للبادرة(جم) تم حسابه ما في نهاية

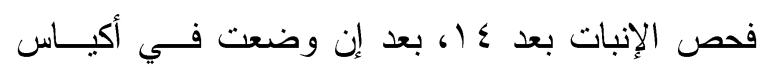
ورقية مثقبة لغرض التجفيف في فرن. . ، درجة مئوية

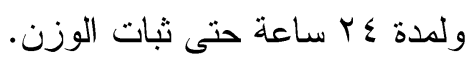

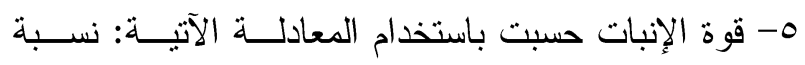

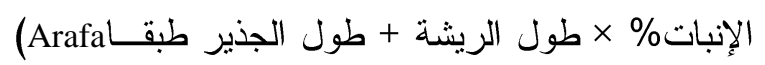

$$
\text { و وآخرون، } 9 \text {. . r). }
$$

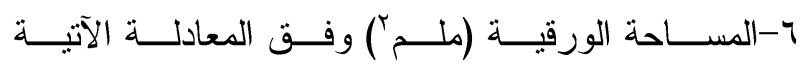

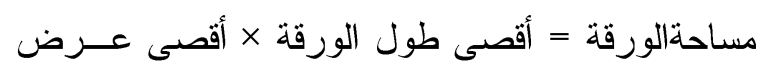

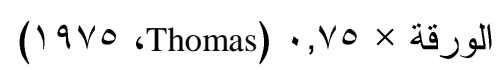

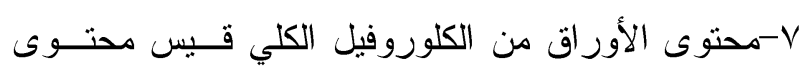

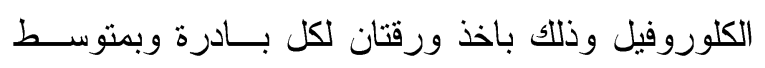

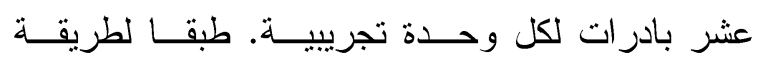
.(19 1 (Machinny)

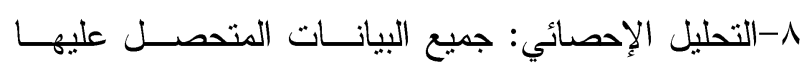

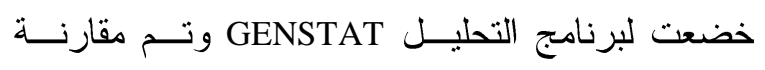

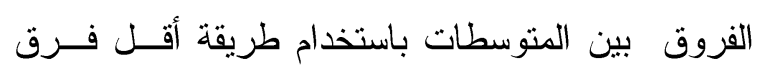

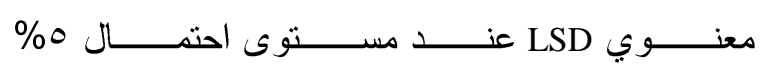
(Gomez and Gomez)) 
جدول اـ تأثير حامض الجبريليك وتركيز ات كلوريد الصوديوم على خصائص الإنبات ونمو وقوة البادرة في الأرة الثامية

\begin{tabular}{|c|c|c|c|c|c|}
\hline طول الجنير) & طول البادرة & قوة الإنبات & نسبة الإببات & في الإنبات الشروم) & المعاملات \\
\hline \multicolumn{6}{|c|}{ الجبريليك } \\
\hline$r, 9 V$ & $1 T, 1 \mathrm{~V}$ & I. & $\leqslant ५, V$ & $7, Y V$ & غير معامل \\
\hline $0, \cdot Y$ & $17,9$. & or. & or,V & $\varepsilon, \varepsilon \vee$ & معامل \\
\hline$\cdot, \wedge \varepsilon$ & $1,1,0$ & 97 & $7 \varepsilon$, & $\cdot, \leqslant \wedge$ & LSD \\
\hline \multicolumn{6}{|c|}{ تركيز كلوريد الصوديوم (جزء بالمليون) } \\
\hline $0,7 \mathrm{~V}$ & r^, VO & 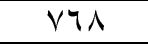 & $\wedge \wedge, r$ & $r, \wedge r$ & 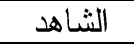 \\
\hline$\neg, \wedge \Gamma$ & 17,01 & $\Lambda I V$ & $\Lambda \cdot, \cdot$ & $\varepsilon, 1 V$ & $7 \ldots$ \\
\hline r, r, & $I V, \ldots$ & 每 & 00, & $0, \pi r$ & $V_{\ldots} \ldots$ \\
\hline$r, \wedge r$ & IY,AT & $1 \wedge r$ & ro, & ( ) & A... \\
\hline$\cdot, \cdots$ & $\cdot, \cdots$ & $\cdot, \cdots$ & $\cdot, \cdot$ & $\mathrm{V}, \mathrm{IV}$ & $9 \ldots$ \\
\hline 1, & $r, V V$ & 101 & $1 \cdot, 1$ & $\cdot, \vee \vee 7$ & $\mathrm{LSD}_{0.05}$ \\
\hline \multicolumn{6}{|c|}{ التفاعل } \\
\hline
\end{tabular}

جدول r . تأثير حامض الجبريليك وتركيزات كلوريد الصوديوم على خصائص الإنبات ونمو وقوة البادرة في الأرة الثامية

\begin{tabular}{|c|c|c|c|c|}
\hline 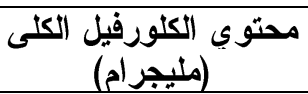 & المساحة الورقية & اللبادرن (الجاف) & الوزن (الغض) للبادرة & المعاملات \\
\hline \multicolumn{5}{|c|}{ الجبريليك } \\
\hline$\cdot, \Gamma \vee \wedge$ & 104. & $\cdot, 1 \vee 7$ & $\cdot, r \cdot r$ & غيرمعامل \\
\hline$\cdot, 7 \leqslant \mu$ & rYo9 & $\cdot, \mathrm{ros}$ & $\cdot, \Sigma\rceil \mu$ & معامل \\
\hline., .01 & $r \leqslant 1$ & $\cdot, \cdot \nabla r$ & $\cdot, 1 \cdot 1$ &. . LSD \\
\hline \multicolumn{5}{|c|}{ تركيز كلوريد الصوديوم (جزء بالمليون) } \\
\hline$\cdot, \wedge 70$ & $01 \cdot \varepsilon$ &., 0 \% & $\cdot, \wedge \wedge \varepsilon$ & 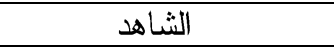 \\
\hline$\cdot, \mathrm{V})$ & YMYI & $\cdot$, TOY & $\cdot, 0 \leqslant 0$ & $7 \ldots$ \\
\hline$\cdot, \leqslant 9 \mu$ & 19.1 & • & $\cdot, \leqslant \wedge$. & $\vee \ldots$ \\
\hline$\cdot, \Sigma \wedge \mu$ & 11.1 & $\cdot$, זצו & $\cdot, \varepsilon \cdot \varepsilon$ & A... \\
\hline$\cdot, \cdots$ & $\cdot, \cdots$ & $\cdot, \cdots$ & $\cdot, \ldots$ & $9 \ldots$ \\
\hline$\cdot, .9 Y$ & 100 &., 110 &., 17. & $\mathrm{LSD}_{0.05}$ \\
\hline \multicolumn{5}{|c|}{ التفاعل } \\
\hline * & ** * & ** & * & \\
\hline
\end{tabular}

r - تأثير تركيز كلوريد الصوديوم

أظهرت النتائج المدونة في جدولي(ا، r) وجود فروقا

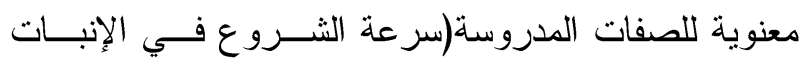

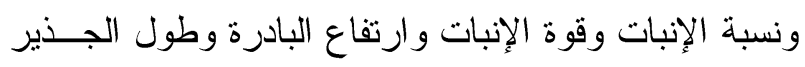

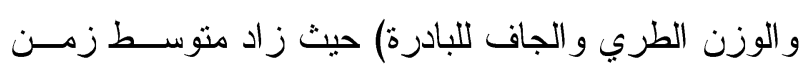

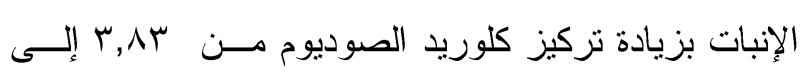
IV,V

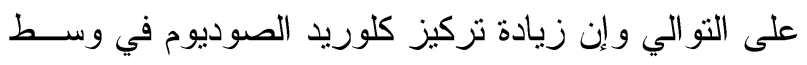

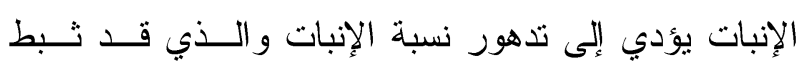

للمساحة الورقية(rVA, · ملجم) للكلورفيل الكلي وقد يرجع ذلك إلى تأثير حامض الجبريليك في زيادة معـدل انقسـام

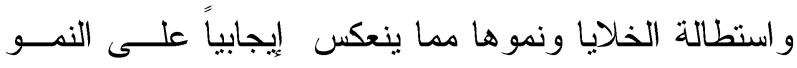

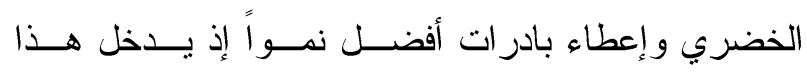
الحامض في تركيب الكلوروفيل فضلاً عن تفوق معساملات النقع بالجبريليك في أسرع شروع للإنبــات ممـــا يعطـيـي

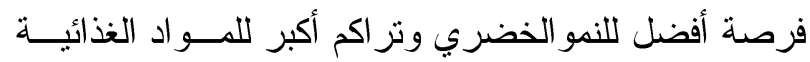
التي تدخل في تركيب مكونات الخلية وهذا يتفق مــع مـــا وجده (Tsakalidi و Barouchas، (1) برحب). 


$$
\text { مجلة الإسكندرية للتبادل العلمى - (مجلد بr العدد () يناير - مارس } 17 \text { • }
$$

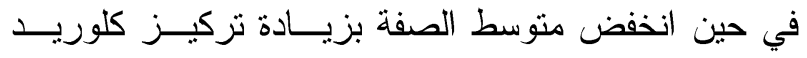

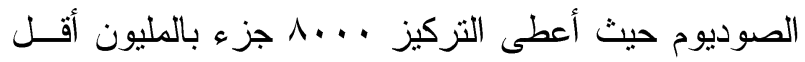

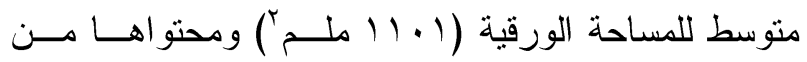

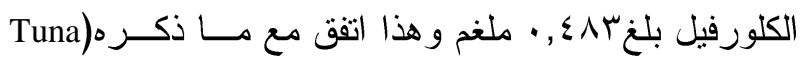

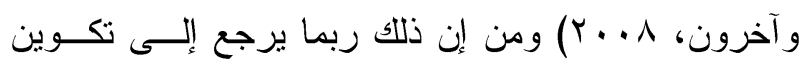

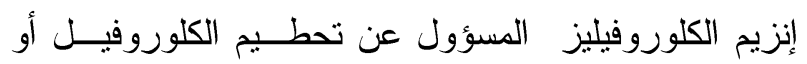
نتيجة التغييرات في تركيب البلاستيدات الخضـــر اء لأوراق

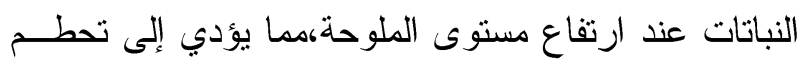
بروتين البلاستيدات واختز ال الكلوروفيل وتثبيط عملية النقل كذلك النقص في البوتاسيوم ودوره الضروري لعملية البناء الضوئي بسبب زيادة نسبة الصوديوم مما يؤدي إلى فقــــان اللون الأخضر وحدوث الاصفر ار للأوراق.

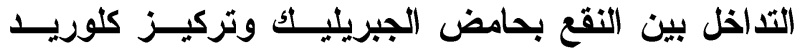

الصوديوم

توضح النتائج المدونة في جدول(ب) تأثير التذاخل بــين

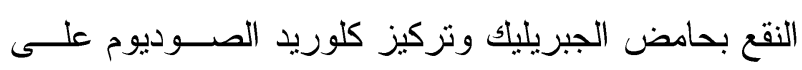
سرعة الانبثاق حيث تفوقت معاملة النقع بحامض الجبريليك

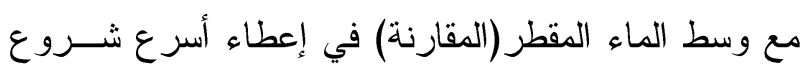

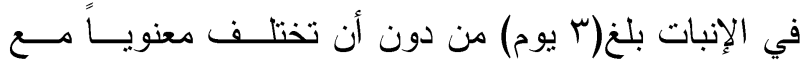
معاملة النقع بحامض الجبريليك عند مستوى ملوحــة . . . جزء بالمليون (سب,r يوم) في حين أعطت معاملة البـذور

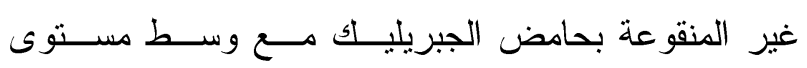

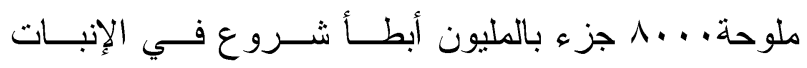
(سب, لايوم) ربما يثير هذا إلى إن نقع البذور بــالتركيزات

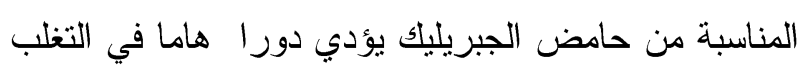

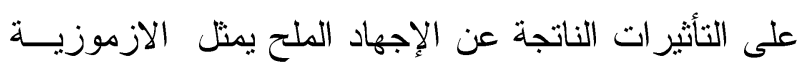

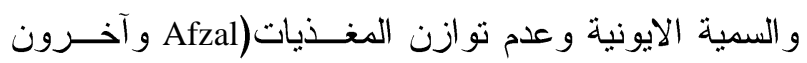
. (r.. o

كذلك نشير البيانات المدونة في جدول(ب) إلـى تــأثير

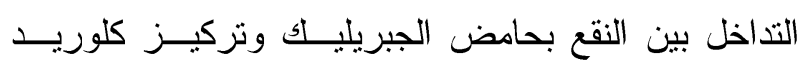
الصوديوم في نسبة وقوة الإنبات إذ تفوقت معاملة النقع
تماماًعندالتركيز · . .9 جزء بالمليون من كلوريد الصوديوم

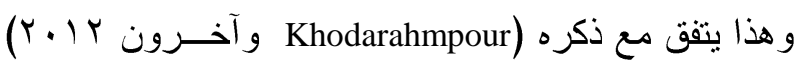
حيث وجد إن ارتفاع الاسموزية لمياه الري يؤدي إلى نقص

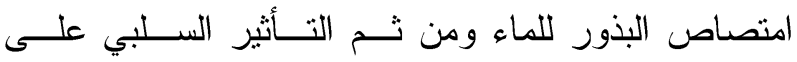
امتصاص المغذيات وتطور الجنين وحدوث السمية الأيونية

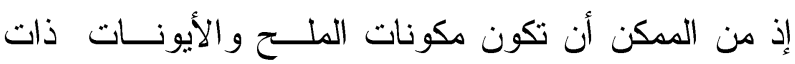
تاثير ات سامة للجنين ولا سيما أيون الصوديوم مما يمنع أو لأ يؤخر الإنبات وسر عته. كما انخفضـــت النســبة المئويــة للإنبات وقوة الإنبات بزيادة نركيز كلوريد الصوديوم فـي

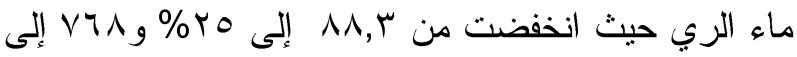

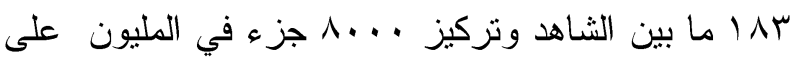

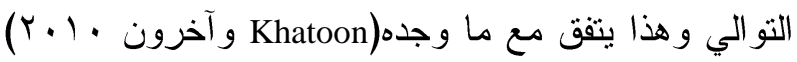

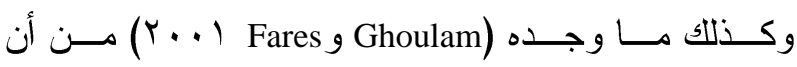

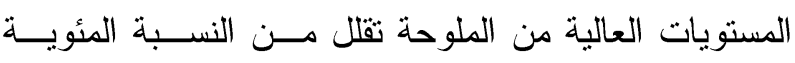

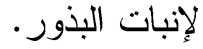
من جهة أخرى كانت اقل القيم لوزن البــادرة الطــري

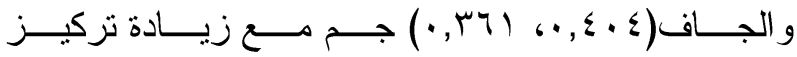
كلوريدالصوديوم في ماء الري إلى . . . . جزء بـــالمليون

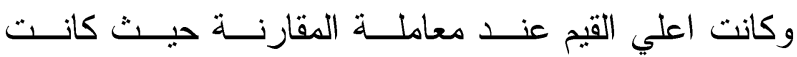

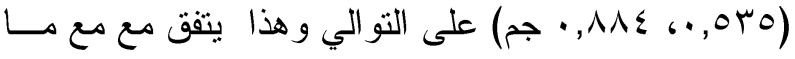

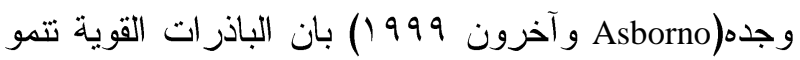
بسرعة وهذا يزيد من الوزن الجاف. كما انخفض ارتفــاع البادرة وطول الجذير بزيادة تركيز الأمسـلاح مسـن (r), TV

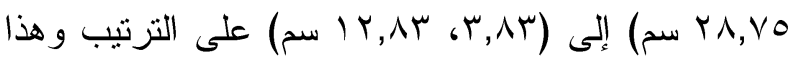

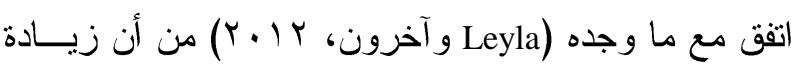

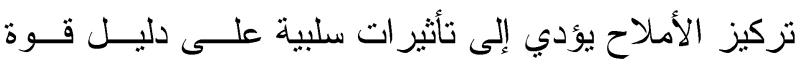

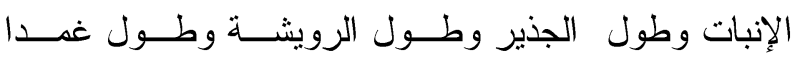
لرويشة.

و على العكس من ذلك تفوقت معاملة الري الماء المقطر

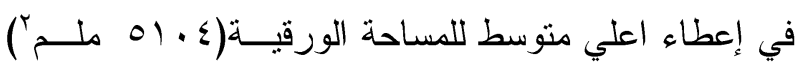

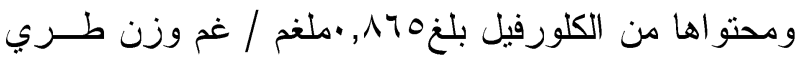


جدول r. تأثير التذاخل بين النقع بحامض الجبريليك وتركيز كلوريد الصوديوم على خصائص الإنبات ونمو وقوة البادرة في الأزرة الثامية

\begin{tabular}{|c|c|c|c|c|c|c|c|c|c|c|}
\hline كلموفيل & المسماحة & الجاتن & الغض (لوزن & الجذيز & البادرة & الإنبات & الإنباًتة & الثُروع فئ الإباتيوم) & (ل) & \\
\hline., 99. & 0990 & - Y १ & $1,1 \mu \mathrm{V}$ & $r, \lambda r$ & $r r, \ldots$ & 910 & $9 \cdot, \cdot$ & $r, \ldots$ & المقارنة & معامل \\
\hline$\cdot, 9 \leqslant Y$ & rTV4 & $\cdot, \leqslant q$. & $\cdot, T \cdot Y$ & $\lambda, r r$ & $\mid \lambda, \lambda \mu$ & 1.10 & $\Delta r, r$ & $r, r \mu$ & $7 \ldots$ & \\
\hline . & 4119 & $\cdot, 0 \leqslant$. & $\cdot, r \cdot \varepsilon$ & $\varepsilon, 9 \pi$ & $1 \wedge, \cdots$ & $\leqslant \leqslant 9$ & $7 \cdot, \cdot$ & $\varepsilon, 7 V$ & $v \ldots$ & \\
\hline$\cdot, 7 \cdot \varepsilon$ & 10.0 & - $2\lceil\uparrow \Lambda$ &.,$r V$. & $0, .$. & $1 \varepsilon, T V$ & r7q & $r_{\cdot, \cdot}$ & $0, \pi$ & $\lambda \ldots$ & \\
\hline$\cdot, \ldots$ & $\cdot, \ldots$ & $\cdot, \ldots$ & $\cdot, \cdots$ & $\cdot, \cdot$, & $\cdot, \cdot$ & $\cdot, \ldots$ & $\cdot, \cdot$ & $\cdot, \cdot$, & $9 \ldots$ & \\
\hline ., $\mathrm{r} r q$ & $\varepsilon r \mid T$ & . & • אד, & $\varepsilon, 0$. & $r \leqslant, O$. & $T Y$. & $\Lambda T, V$ & $\varepsilon, T V$ & الدقارنة & \\
\hline.,$\varepsilon \vee \wedge$ & $11 \leqslant$. & $\cdot, r) \leqslant$ & $\cdot, \varepsilon \wedge \mathrm{V}$ & $0, r \mu$ & $I \varepsilon, \Gamma \mu$ & 711 & $V Y, V$ & $0, \ldots$ & $7 \ldots$ & معامل \\
\hline$\cdot, r \cdot q$ & $17 . Y$ &., $19 \mathrm{~V}$ &., 104 & $r, r r$ & $17, \ldots$ & TIV & $0 ., \cdot$ & $7, \ldots$ & $v \cdots$ & \\
\hline צדז,. & 797 & $\cdot, r q \varepsilon$ &.,$Y \mu \lambda$ & $Y, T V$ & $11, \ldots$ & $9 \mathrm{~V}$ & $r \cdot, \cdot$ & $V, r r$ & $\lambda \ldots$ & \\
\hline$\cdot, \ldots$ & $\cdot, \ldots$ & $\cdot, \ldots$ & $\cdot, \ldots$ & $\cdot, \cdot$, & $\cdot$, & $\cdot, \cdot$ & $\cdot$, & $\cdot, \cdot$. & $9 \ldots$ & \\
\hline., $1 \tau$. & $1 r .9$ & . &.,$Y Y Y$ & $1, \wedge \wedge$ & $r, 9 r$ & $r \backslash \varepsilon$ & $1 \varepsilon, r$ & $1, \cdot 1$ & LSD & \\
\hline
\end{tabular}

أعطت معاملة الغير منقوعة في حامض الجبريليك وتركيز

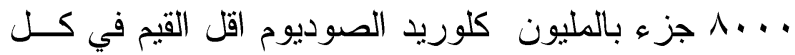

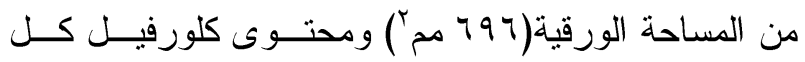

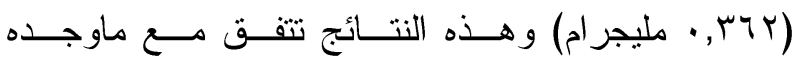
Ashraf و Iqbal) أدت إلى ارتفاع محتوى الكلوروفيل لبادرات الذرة الثــامية النامية تحت تأثير الإجهاد الملحي.

\section{المــــ}

أبوزيــد، الثــــات ـ99 19: الهرمونـــات النباتيـــة و التطبيقــات الزر اعيــة، مؤسســـة عز الــدين للطباعــة و النثــــ، القاهرة

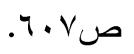

Afzal, I., M. A. Basra, and I.Amir. 2005. The effect of seed soaking with plant growth regulators on seedling vigor of wheat under salinity strees. Journal of Stress Physiology and Biochemistry. 1(1): 6-14.

AOSA, (Association of Official Seed Analysts). 1983. Seed Vigour Testing Handbook.Contribution No. 32 to Handbook on Seed Testing Association of Official Seed Analysts, Lincoln, NE, USA. pp. 88

Arafa, A.A., M. A. Khafagy, and M. F. El-Banna. 2009. The effect of glycine betaine or ascorbic acid on grain germination and leaf structure of sorghum plant grown under salinity stress. J. Crop Sci. 3 (5): 294-304.

Asborno, M.D., A.A. Vidai, R. Bezus, and J. Beltrano. 1999. Rice temperature and gibberellic acid effect on initial growth stages. Agro - ciencia , 15 (1): 47-53.
بحامض الجبريليك مع وسط الماء المقطر في إعطاء اعلي

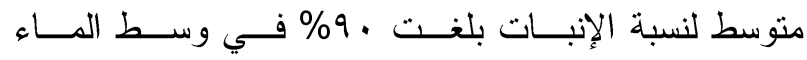

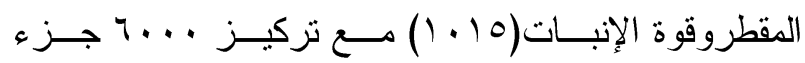

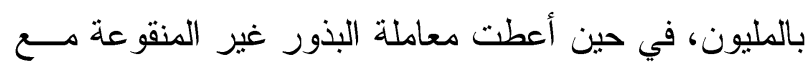

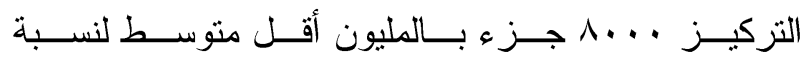
الإنبات ·r\% قوة الإنبات(9V) ربما يعود ذلك إلى إن نقع

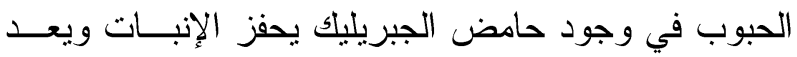

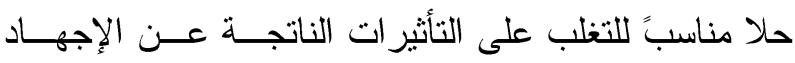

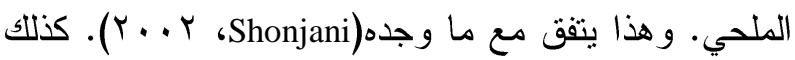
فان بيانات جدول(ץ) نثير إلى تأثير التداخل بين عـاملي

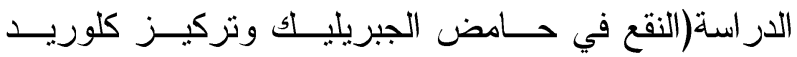
الصوديوم) على نمو البادرة من حيث الارتفــاع و الــوزن الجاف إذا عطت معاملة النقع بحامض الجبريليك في كـلـل

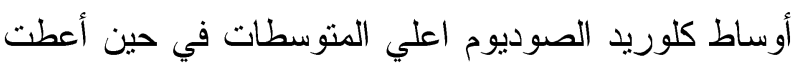
الغير منقوعة في حامض الجبريليك اقل المتوسطات و وهذا

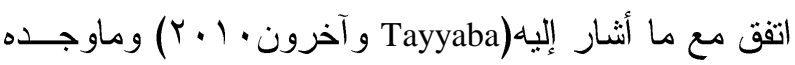
Leyla)

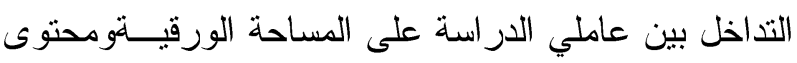
الكلوروفيل إذ أعطت معاملة النقع بحامض الجبريليك فـي لكي

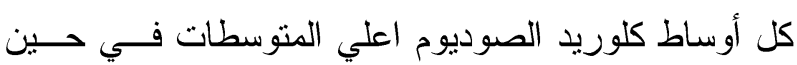




$$
\text { مجلة الإسكندرية للتبادل العلمى - (مجلد Vr العدد () يناير - مارس ب } 1 \text {. }
$$

Leyla, I., Z. Dumlupinar, S. N. Kara, C. Yururdurmaz, and M. Cölkesen. 2012. The effect of different temperatures and salt concentrations on some popcorn lium). AJCS., 5(8): 973-978

Machinny, G., 1941 . Absorption of light by chlorophyll solution. J. Biol Chem., 140: 315- 322 .

Majid, K., and R. Gholamin. 2011. Effects of salt stress levels on five maize (Zea mays L.) cultivars at germination stage. Afric J. Biotech, 10(60): 12909-12915 sen.pp.164.

Shonjani, S. 2002. Salt sensitivity of Rice, Maize, Sugar Beet, and Cotton during germ- ination and early vegetative Growth.Ph.D. Dissoretion, Justus Liebig University Giessen.pp.164.

Tanji, K. K. 2004. Salinity in the Soil in Salinity Environment - Plants- Molecules, A. Lauchli and L. Lütteg (eds.), Kluwer academic publishers, Dordrecht.pp.552.

Tayyaba, K., K. Hussain, A. Majeed, K. Nawaz, and M. F. Nisar. 2010. Morphological variations in maize (Zea mays L.) underdifferent levels of $\mathrm{NaCl}$ at germinating stage. World Appl. Sci. J., 8(10): 1294-1297.

Thomas, H. 1975. The growth response of weather of simulated vegetative swards of single genotype of Lolium perenne. J. Agric. Sci. Camb. 84 : 333-343.

Tsakalidi, A. L., and P. E. Barouchas.2011. Salinity, chitin and GA3 effects on seed germination of chervil (Anthriscus cerefo- lium).AJCS., 5(8): 973-978.

Tuna, A., C. Kaya, M. Diklitas, and D. Higgs. 2008. The combined effects of gibbere llic acid and salinity on some antioxidant enzyme activities, plant growth parameters and nutritional status in maize plants. Environmental and Experimental Botany., 62: 1-9.
Attiya, H.J., and K.A.Jaddoa. 2011. Plant Growth Regulater, The Theory and Practice. Ministry of Higher Education and Scientific Research.Publication Republic of Iraq.

Carpici, E. B., N. Celik, and G. Bayam. 2009 . Effect of salt stress on germination of some maiza (Zea mays L.) cultivars. Afr. J. Biotechnology. 8(19): 4918-4922.

FAO. 2000. Global Network on Integrated Soil Management Sustainable Use of Salt Effected Soils. Available in: ttp://www.Faw.org/ag/AGL/agll/spush/intro.

Ghoulam, C., and K. Fares. 2001. Effect of salinity on seed germination and early seedling growth of sugar beet.Seed Sci.Tech. 29: 357-364.

Gomez, K.A., and A.A. Gomez. 1984. Statistical Procedure for Agricultural Research.John Wiley and Sons.Now York,usA.

Hussain. K., M. F. Nisar, A. Majeed, K. Nawaz, K. H. Bhatti, S. Afghan, A. Shahazad, and S. Z. Hussnian. 2010. What molecular mechanism is adapted by plants duringsalt stress tolerance. Afric J. Biotech. 9(4): 416-422.

Iqbal, M., and M. Ashraf. 2010. Gibberellic acid mediated induction of salt tolerance in wheat plants: Growth, ionic partitioning, pho- tosynthesis, yield and hormonal homeosta-sis. Environmental and Experimental Botany.pp.10.

ISTA (International Seed Testing Association). 2005. International Rules for Seed Testing. Adopted at the Ordinary Meeting. 2004, Budapest, Hungary to become effective on 1st January 2005.The International Seed Testing Association. (ISTA).

Khodarahmpour, Z., M. Ifar, and M. Motamedi. 2012. Effects of $\mathrm{NaCl}$ salinity on maize (Zea mays L.) at germination and early seedling stage.Africa J. Biotech.11 (2): 298-304. 


\section{ABSTRACT \\ Effect of GA3 on Germination Characteristics and Seedling Growth under Salt Stress Conditions in Maize (Zea mays L)}

Fatma A. Faraj, Ahmed. S.Buhedma

An experiment was conducted in Cereal Technology Lab-Faculty of Agriculture- Omar, ELMokhtar University- A factorial experiment was conducted according to completely randomize design with three replications, during 2015 season with two factors in order to study the effect of soaking seeds in gibberellic acid on germination and growth of seedling maize (v. 310) under salt stress conditions. First treatment was soaked seeds in $500 \mathrm{ppm}$ gibberllic acid for 24 hours Vs non soaked. Second treatment was different concentration of $\mathrm{NaCl}$ (zero, 6000, 7000, 8000, 9000 $\mathrm{ppm})$. The results showed the superiority of seeds soaked in gibberellic acid in giving faster germination start, the highest percentage of germination, fresh and dry weight seedling vigor, seedling length and chlorophyll content, As for the concentrations of $\mathrm{NaCl}$, the planting media distilled water (Control) gave the lowest averages for faster start while low germination percentage was noticed when the concentrations of $\mathrm{NaCl}$ increased. The results also showed significant interaction effect between soaked treatment in gibberellic acid and $\mathrm{NaCl}$ concentration in most treatments. Increasing sodium chloride concentration in germination media greatly reduced germination percentage and seed germination completely inhibited at $9000 \mathrm{ppm} \mathrm{NaCl}$. The results of this study recommend soaking maize seeds in $500 \mathrm{ppm}$ gibberellic acid for 24 hours before planting, especially in salt affected soils.

Key words: Salinity, Seed vigor, Germination time, Gibberellic acid, Zea mays $L$. 